\title{
Estudo comparativo de idosos ativos e inativos através da avaliação das atividades da vida diária e medição da qualidade de vida
}

\author{
Comparative study of active and inactive elderly persons through the \\ assessment of activities of daily living and quality of life
}

\author{
S. Caporicci, M.F.O. Neto
}

RESUMO

Este trabalho teve como objetivo avaliar as atividades da vida diária e a qualidade de vida de idosos ativos e inativos. Caracterizou-se como uma pesquisa descritiva de caráter quantitativo. Participaram da pesquisa 24 idosos, sendo 12 praticantes de atividade física (PAF) e 12 não praticantes (NPAF). Foram utilizados como instrumentos, um questionário sociodemográfico, o SF-36 para a medição da qualidade de vida e os testes propostos pelo GDLAM. Para análise dos dados utilizamos o programa estatístico SPSS 15.0. As médias dos resultados obtidos pelo questionário sociodemográfico, para os grupos PAF e NPAF, respectivamente foram: idade - 69.5 e 70.0 anos; estado civil - $50.0 \%$ viúvo e $41.7 \%$ casados; escolaridade $-50.0 \%$ com $1^{\circ}$ grau completo e $58.3 \%$ com $1^{\circ}$ grau incompleto; problemas de saúde $-25.0 \%$ e $58.33 \%$ com hipertensão. No questionário SF-36, ambos os grupos apresentaram qualidade de vida similares, embora o grupo PAF apresentasse tendência a escores mais altos. Em todos os testes de autonomia funcional, o grupo PAF apresentou tendência a um menor tempo de execução quando comparados com o grupo NPAF; entretanto, ambos os grupos encontraram-se na classificação "fraco" de acordo com o protocolo GDLAM. Concluímos que não houve diferença estatisticamente significativa entre os grupos.

Palavras-chave: idosos, qualidade de vida, autonomia funcional

ABSTRACT

This study aimed at evaluating the activities of daily living and quality of active and inactive seniors' life. This is a descriptive research of a quantitative nature. Participants were 24 seniors, 12 physical activity practitioners (PPA) and 12 non-practitioners (NPPA). The instrument was a demographic questionnaire and SF-36 for measuring the quality of life and tests proposed by GDLAM. SPSS 15.0 was used for data analysis. The results obtained by the sociodemographic questionnaire for PPA and NPPA groups were, respectively: age -69.5 and 70.0 years, marital status $-50.0 \%$ widower and $41.7 \%$ were married; education $-50.0 \%$ with complete elementary school and $58.3 \%$ with incomplete elementary school; health problems $-25.0 \%$ and $58.33 \%$ hypertension. In functional autonomy tests, the PPA group had shorter execution time when compared with the NPPA group; however, both groups found themselves in the rating of "weak" according to the protocol GDLAM. We concluded that there was no statistical difference between groups.

Keywords: elderly, quality of life, functional autonomy

Submetido: 21.02.2010 | Aceite: 04.07.2010

Sarah Caporicci e Manoel Freire de Oliveira Neto. Grupo de Pesquisa em Envelhecimento, Saúde e Motricidade Humana - GPESAM; Departamento de Educação Física - DEF, Universidade Estadual da Paraíba - UEPB, Brasil.

Endereço para correspondência: Sarah Caporicci, Rua Vigário Virgínio, 417, Ap. 106., Santo Antônio - Campina Grande, CEP: 58103-340 Paraíba - PB, Brasil.

E-mail: sarah_caporicci@hotmail.com 
O crescimento no número de idosos e o aumento da expectativa de vida mundial têm gerado uma maior preocupação quanto a um envelhecer mais saudável, independente, ativo e com qualidade. Atualmente, uma em cada dez pessoas no mundo tem 60 anos ou mais e as estimativas para o ano de 2050, é de uma para cada cinco, aumentando de uma para três nos países desenvolvidos (IBGE, 2002).

$\mathrm{O}$ processo de envelhecimento pode ser determinado pela perda funcional progressiva que ocorre de forma gradual, universal e irreversível com o avançar da idade (Nahas, 2006).

Apesar da limitação da idade cronológica de 60 anos como marco inicial da velhice, muitas vezes este marco não corresponde à idade biológica do indivíduo (Mazo, Lopes, \& Benedetti, 2004). Assim, o processo de envelhecimento não pode ser definido apenas pela sua idade cronológica; outros fatores como as condições físicas, funcionais, mentais, de saúde, entre outros, podem influenciar diretamente sobre este processo, o que caracteriza que o envelhecimento é algo individualizado que difere de pessoa para pessoa.

O envelhecimento caracteriza-se pelo declínio das funções orgânicas e inicia-se por volta dos 30 anos de idade, onde a cada ano, acredita-se que há uma perda de $1 \%$ da função. O processo de envelhecimento é comum a todos, porém, o ritmo em que ocorre este declínio varia não apenas de um órgão para outro, mas de idoso para idoso com a mesma idade cronológica (Netto \& Ponte, 2005). De acordo com Santos e Knijnik (2006), a idade biológica de indivíduos fisicamente ativos pode ser diminuída entre 10 a 20 anos quando comparados com idosos fisicamente inativos.

O decréscimo da capacidade funcional é provocado, em grande parte, pelo desuso procedente do sedentarismo, o que pode ser melhorada pela prática regular de exercícios ou adoção de um estilo de vida mais ativo, protelando os efeitos nocivos causados pelo processo de envelhecimento (Nahas, 2006), e auxiliando no tratamento ou controle de doenças crónico-degenerativas, manutenção das funções locomotivas, melhor desempenho na realização das atividades da vida diária e um maior grau de independência e autonomia, permitindo que o idoso permaneça ativo e com uma boa qualidade de vida (Okuma, 1998).

A capacidade funcional está relacionada com a propensão de uma pessoa realizar as atividades da vida diária (AVDs) de maneira independente (Matsudo, 2002). Conforme o aumento da idade cronológica há uma tendência das pessoas serem menos ativas, diminuindo a sua capacidade funcional, aumentando, assim, o seu grau de dependência para a realização de suas atividades básicas, tais como vestir-se, calçar os sapatos, escovar os dentes, tomar banho, alimentar-se, entre outros (Vale, 2004).

A realização das AVDs é um dos fatores que determinam a expectativa de vida ativa do idoso, sendo de grande importância sua aplicabilidade para determinar seu nível de independência e autonomia. Nesta perspectiva, este trabalho teve como objetivo avaliar as atividades da vida diária e a qualidade de vida de idosos ativos e inativos, através da comparação da capacidade funcional em relação às atividades da vida diária (AVDs), utilizando o protocolo de avaliação da autonomia funcional (GDLAM) e da medição da qualidade de vida relacionada à saúde de idosos, utilizando o instrumento SF-36.

\section{MÉTODO}

Este estudo caracterizou-se como uma pesquisa descritiva de caráter quantitativo.

\section{Amostra}

Este estudo teve como tipo de amostra nãoprobabilista intencional, sendo constituída por 24 idosas, com idades a partir de 60 anos, sendo 12 praticantes e 12 não praticantes de atividades físicas, participantes do grupo "Anos Dourados" da Pastoral do Idoso no bairro de Bodocongó em Campina Grande - PB. As idosas praticantes de atividade física (PAF) 
realizavam aulas de ginástica aeróbica, oferecidas no próprio local, com frequência de três vezes semanais, e o grupo de não praticantes de atividade física (NPAF) relataram não praticar nenhum tipo de atividade física sistematizada.

Participaram da pesquisa, todas as idosas a partir de 60 anos presentes no dia da aplicação dos questionários e testes, praticantes e não praticantes de atividades físicas com condições de saúde satisfatória para realizar a bateria de testes.

\section{Instrumentos}

Foi aplicado um questionário sociodemográfico, um questionário para a medição da qualidade de vida relacionado à saúde, o SF36.

O Medical Outcomes Study 36-item Short-Form Health Survey (SF-36), foi traduzido, adaptado e validado para a cultura brasileira, sendo este utilizado para avaliar a qualidade de vida tanto da população em geral quanto de idosos (Ciconelli, 1997). O instrumento consta de 36 itens subdivididos em 8 dimensões: função física, rol físico, dor corporal, saúde geral, vitalidade, função social, rol emocional e saúde mental. Como parâmetro de pontuação da média do questionário SF-36, utilizamos como valores de referência a tabela abaixo.

Tabela 1

Classificação da pontuação transformada na escala de o a 100, obtidas através do questionário SF-36 (Adaptado de URSS, 2000)

\begin{tabular}{cc}
\hline Classificação & Escala de 0 a 100 \\
\hline Excelente & 100 \\
Muito Boa & 84 \\
Bom & 61 \\
Regular & 25 \\
Ruim & 0 \\
\hline
\end{tabular}

Para mensurar a autonomia funcional dos idosos, foi utilizada uma bateria de testes de acordo com o protocolo do Grupo de Desenvolvimento Latino-Americano para a
Maturidade (GDLAM): caminhada $10 \mathrm{~m}$ (C10M), levantar-se da posição de decúbito ventral (LPDV), sentar-se, levantar-se da cadeira e locomover-se (LCLC) e levantar-se da posição sentada (LPS) (Dantas, Vale, \& Pernambuco, 2004). Os testes são destinados a indivíduos fisicamente ativos, sendo de extrema importância, na medida em que é capaz de detectar níveis de capacidade funcional e ser utilizada para avaliação do desenvolvimento de indivíduos inseridos em programas de atividades físicas.

Os equipamentos utilizados para os testes são descritos a seguir:

- C10M: para este procedimento utilizaramse uma trena, para precisar a distância, e um cronômetro, para marcar o tempo despendido na atividade;

- LPDV: utilizaram-se um colchonete e um cronômetro para medir o tempo gasto na atividade;

- LCLC: para este teste foi utilizada uma cadeira de braços com altura entre 43 e $50 \mathrm{~cm}$, uma trena, dois cones, um cronômetro e fita adesiva;

- LPS: utilizam-se neste teste uma cadeira com altura entre 43 e $50 \mathrm{~cm}$ e um cronômetro.

\section{Procedimentos}

Os questionários sociodemográfico e o de qualidade de vida relacionada à saúde SF-36 foram aplicados individualmente, sem prévia ajuda ou indução das respostas dos mesmos.

Em seguida foram realizados individualmente os testes de acordo com o protocolo GDLAM: caminhada $10 \mathrm{~m}$ (C10M), levantar-se da posição de decúbito ventral (LPDV), sentar-se, levantar-se da cadeira e locomover-se (LCLC) e levantar-se da posição sentada (LPS) (Dantas et al., 2004). Pelos resultados obtidos em segundos, verificou-se a dependência ou independência dos idosos nas AVDs.

Na Tabela 2 são apresentados os valores de referência para os vários testes de avaliação da autonomia funcional. 
18 | S. Caporicci, M.F.O. Neto

Tabela 2

Níveis de classificação de avaliação da autonomia funcional do protocolo GDLAM

\begin{tabular}{cccccc}
\hline & \multicolumn{5}{c}{ Testes } \\
Classificação & C10M (s) & LPS (s) & LPDV (s) & LCLC (s) & IG (escore) \\
\hline Fraco & $>7.09$ & $>11.19$ & $>4.40$ & $>43.00$ & $>28.54$ \\
Regular & $7.08-6.34$ & $11.18-9.55$ & $4.39-3.30$ & $42.99-38.69$ & $28.53-25.25$ \\
Bom & $6.33-5.71$ & $9.54-7.89$ & $3.29-2.63$ & $38.67-34.78$ & $25.24-22.18$ \\
Muito Bom & $<5.70$ & $<7.88$ & $<2.62$ & $<34.77$ & $<22.17$ \\
\hline
\end{tabular}

As idosas iniciaram os testes após o sinal dado pelo avaliador, que aferiu em segundos o tempo gasto na realização de cada teste. Os dados coletados foram utilizados para classificar os grupos de acordo com os padrões da GDLAM (Dantas et al., 2004) e para estabelecer o índice geral de autonomia (IG). O IG foi calculado de acordo com a fórmula descrita a seguir:

$$
\begin{gathered}
\mathrm{IG}=\frac{[(\mathrm{C} 10 \mathrm{~m}+\mathrm{LPS}+\mathrm{LPDV}) \times 2]+\mathrm{LCLC}}{3} \\
\text { Onde }=\text { C10 m, LPS, LPVD e LCLC = tempo aferido em } \\
\text { segundos; IG = indice GDLAM }
\end{gathered}
$$

Teste 1 - Caminhar 10 metros (C10M): O propósito deste teste é avaliar a velocidade que o indivíduo leva para percorrer a distância de 10 metros (Sipila, Multanen, Kallinen, Era, \& Suominen, 1996).

Teste 2 - Levantar-se da posição sentada (LPS): O teste visa avaliar a capacidade funcional da extremidade inferior e consiste em: o indivíduo, partindo da posição sentada em uma cadeira, sem apoio dos braços, estando o assento a uma distância do solo de $50 \mathrm{~cm}$, levanta-se e senta-se cinco vezes, consecutivamente (Guralnik et al., 2000).

Teste 3 - Levantar-se da posição decúbito ventral (LPDV): O propósito deste teste é avaliar a habilidade do indivíduo para levantarse do chão. O teste consiste em: partindo da posição inicial em decúbito ventral, com os braços ao longo do corpo, ao comando de "já", o indivíduo deve levantar-se, ficando em pé o mais rápido possível (Alexander, Ulbrich, Raheja, \& Channer, 1997).

Teste 4 - Levantar-se da cadeira e locomover-se pela casa (LCLC): O objetivo é avaliar a capacidade do idoso na sua agilidade e equilíbrio, em situações da vida. Com uma cadeira fixa no solo, deve-se demarcar dois cones diagonalmente à cadeira, a uma distância de quatro metros para trás e três metros para os lados direito e esquerdo da mesma. O indivíduo inicia o teste sentado na cadeira, com os pés fora do chão, e ao sinal de "já", ele se levanta, move-se para direita, circula o cone, retorna para a cadeira, senta-se e retira ambos os pés do chão. Sem hesitar, faz o mesmo movimento para a esquerda. Imediatamente, realiza novo percurso, para a direita e para a esquerda, assim perfazendo todo o percurso e circulando cada cone duas vezes, no menor tempo possível (Andreotti \& Okuma, 1999).

\section{Análise Estatística}

Os dados foram analisados mediante análise descritiva, através do programa SPSS 15.0, utilizando o Teste $\mathrm{F}$ - de Snedcor ( $F$ test) para comparação das médias pela análise de variância (ANOVA one-way) para comparação entre os grupos etários, da medição da qualidade de vida e capacidade funcional, adotando o intervalo de confiança de $95 \%$. Os valores foram considerados significativos quando $p<.05$. Para as demais análises foram utilizadas como parâmetros, as percentagens, as médias e os desvios padrão. 


\section{RESULTADOS}

\section{Aspectos sociodemográficos}

De acordo com a Tabela 3, todos os idosos que frequentavam o grupo de convivência eram do gênero feminino. A análise estatística não mostrou diferença significativa de idade entre os grupos $\left(F_{(1,22)}=.034, p>.05\right)$.

Tabela 3

Características sociodemográficas dos idosos praticantes (PAF) e não praticantes de atividade física (NPAF)

\begin{tabular}{lcc}
\hline \multicolumn{1}{c}{ Grupos } & NPAF & PAF \\
\hline Idade & $70.0 \pm 6.6$ & $69.5 \pm 6.7$ \\
Estado Civil & & \\
Solteiro & $8.3 \%$ & $8.3 \%$ \\
Casado & $41.7 \%$ & $33.3 \%$ \\
Viúvo & $33.3 \%$ & $50.0 \%$ \\
Separado & $16.7 \%$ & $8.3 \%$ \\
Escolaridade & & \\
$1^{\circ}$ Grau Incompleto & $58.3 \%$ & $16.7 \%$ \\
$1^{\circ}$ Grau & $25.0 \%$ & $50.0 \%$ \\
$2^{\circ}$ Grau & $16.7 \%$ & $33.3 \%$ \\
Problemas de Saúde & & \\
Nenhum & $25.0 \%$ & $25.0 \%$ \\
Cardíaco & $16.7 \%$ & $0.0 \%$ \\
Respiratório & $8.3 \%$ & $8.3 \%$ \\
Articular & $33.3 \%$ & $25.0 \%$ \\
Hipertensão & $58.3 \%$ & $25.0 \%$ \\
Diabetes & $8.3 \%$ & $8.3 \%$ \\
Osteoporose & $25.0 \%$ & $16.7 \%$ \\
Outros & $16.7 \%$ & $25.0 \%$ \\
\hline
\end{tabular}

Em relação ao estado civil, observa-se que no grupo NPAF a maior percentagem foi de idosos casados $(41.7 \%)$, seguidos de viúvos (33.3\%), separados (16.7\%) e solteiros $(8.3 \%)$, enquanto no grupo PAF a maior percentagem foi de viúvos $50.0 \%$, seguidos de casados (33.3\%) e separados e solteiros (8.3\%, cada). Para a escolaridade, a percentagem maior obtida para os idosos NPAF foi de $58.3 \%$ para $1^{\circ}$ grau incompleto, seguida de $25.0 \%$ para $1^{\circ}$ grau e $16.7 \%$ para $2^{\circ}$ grau. Já no grupo de idosos PAF, a maior percentagem obtida foi de
$50.0 \%$ para $1^{\circ}$ grau, seguida de $33.3 \%$ para $2^{\circ}$ grau e $16.7 \%$ para $1^{\circ}$ grau incompleto. Em relação aos problemas de saúde, em ambos os grupos estudados, $25.0 \%$ dos idosos relataram não ter nenhum problema de saúde. Dentre os idosos que apresentavam um ou mais problemas, no grupo NPAF, $58.3 \%$ sofriam de hipertensão, $33.3 \%$ de problemas articulares, $25.0 \%$ de osteoporose e $16.7 \%$ de problemas cardíacos. Com relação ao grupo PAF, houve uma menor prevalência nas doenças relacionadas, onde $25.0 \%$ relataram ter hipertensão, problemas articulares ou outros, e $16.7 \%$ osteoporose.

\section{Medição da qualidade de vida através do Questionário SF-36}

De acordo com a Tabela 4, nos diferentes domínios do questionário de qualidade de vida relacionado à saúde SF-36, para os praticantes de atividade física (PAF), podemos destacar as maiores pontuações nos seguintes domínios: Função física (68.16 \pm 26.78), refere-se ao grau em que a falta de saúde limita as atividades físicas e atividades da vida diária; Rol emocional $(66.66 \pm 40.20)$ enfoca o grau em que os problemas emocionais interferem no trabalho ou atividades diárias; e, Rol físico $(62.50 \pm 37.68)$, grau em que a falta de saúde interfere no trabalho e outras atividades diárias. A média mais baixa obtida neste escore foi o domínio Dor corporal (33.33 \pm 23.86$)$, que corresponde à intensidade de dor que influencia nas atividades da vida diárias.

Em relação às pontuações médias obtidas no grupo de não praticantes de atividade física (NPAF), podemos destacar as maiores pontuações, no domínio Função física (62.06 $\pm 27.25)$, Rol emocional $(61.11 \pm 44.57)$ e Rol físico $(60.40 \pm 37.62)$. A média mais baixa obtida neste escore foi o domínio Dor corporal (35.83 \pm 25.03$)$. A análise estatística revelou que não houve diferença estatisticamente significativa entre os grupos, para todos os domínios. 
Tabela 4

Pontuação transformada (0-100) obtida do questionário de qualidade de vida SF-36 para os grupos PAF e NPAF

\begin{tabular}{ccccccccc}
\hline & Função & Rol & Dor & Saúde & & Função & Rol & Saúde \\
& Física & Físico & Corporal & Geral & Vitalidade & Social & Emocional & Mental \\
& $M(D P)$ & $M(D P)$ & $M(D P)$ & $M(D P)$ & $M(D P)$ & $M(D P)$ & $M(D P)$ & $M(D P)$ \\
\hline \multirow{2}{*}{ PAF } & 68.16 & 62.50 & 33.33 & 53.75 & 50.83 & 43.75 & 66.66 & 56.33 \\
& $(26.78)$ & $(37.68)$ & $(23.86)$ & $(14.32)$ & $(13.95)$ & $(13.58)$ & $(40.20)$ & $(7.52)$ \\
& 62.06 & 60.40 & 35.83 & 47.08 & 48.75 & 42.70 & 61.11 & 53.33 \\
NPAF & $(27.25)$ & $(37.62)$ & $(25.03)$ & $(9.87)$ & $(8.01)$ & $(14.55)$ & $(44.57)$ & $(11.60)$ \\
& .52 & .89 & .80 & .19 & .65 & .85 & .75 & .46 \\
\hline
\end{tabular}

Nota: PAF - praticantes de atividade física; NPAF - não praticantes de atividade física

Tabela 5

Análise descritiva, em segundos, dos grupos PAF e NPAF de acordo com o protocolo GDLAM

\begin{tabular}{cccccc}
\hline & C10M (s) & LPS (s) & LPDV (s) & LCLC (s) & IG (escore) \\
& $M \pm D P$ & $M \pm D P$ & $M \pm D P$ & $M \pm D P$ & \\
\hline PAF & $7.91 \pm 1.35$ & $12.51 \pm 3.52$ & $5.45 \pm 2.54$ & $46.89 \pm 8.08$ & 31.92 \\
(Classificação) & $($ Fraco $)$ & (Fraco) & (Fraco) & $($ Fraco) & $($ Fraco) \\
NPAF & $9.40 \pm 3.55$ & $19.10 \pm 13.69$ & $11.02 \pm 9.42$ & $55.35 \pm 17.28$ & 37.88 \\
(Classificação) & $($ Fraco $)$ & (Fraco) & (Fraco) & (Fraco) & $($ Fraco) \\
$p$ & .23 & .15 & .08 & .17 & -
\end{tabular}

Nota: PAF - praticantes de atividade física; NPAF - não praticantes de atividade física; C10M - corrida de 10 metros; LPS levantar-se da posição sentada; LPDV - levantar-se da posição decúbito ventral; LCLC - levantar-se da cadeira e locomoverse pela casa; IG - índice GDLAM.

\section{Avaliação da autonomia funcional através dos testes GDLAM}

De acordo com a Tabela 5, referente aos resultados da avaliação da autonomia funcional dos idosos utilizando o protocolo (GDLAM), o grupo PAF apresentou melhores tempos na realização de todos os testes em relação ao grupo NPAF, sugerindo que o primeiro grupo tende a apresentar uma melhor mobilidade na realização das atividades diária em relação ao segundo grupo. Entretanto, ambos os grupos apresentam um baixo nível de capacidade funcional de acordo com o protocolo GDLAM, enquadrando-se na classificação "fraco" em todos os testes realizados e não existindo diferenças estatisticamente significativas entre os grupos $(p>.05)$.

\section{DISCUSSÃO}

De acordo com as características sociodemográficas, estudos comprovam a prevalência do género feminino em grupos de convivência ou grupos da terceira idade verificando que a participação masculina raramente ultrapassa 20\% (Gonçalves, Dias, \& Liz, 1999). Conforme o estudo de Santos, Santos, Fernandes e Henriques (2002a), a predominância do género feminino foi de $79.7 \%$ enquanto o género masculino foi de $20.3 \%$. Observa-se que as mulheres predominam em grupos de convivência e de prática de atividades físicas, fazendo-se necessário uma maior intervenção e incentivo das políticas públicas, dos profissionais de saúde e da família para inserir os homens nestes programas.

Em concordância com o estudo de Santos et al. (2002), os viúvos predominaram com $41.4 \%$, seguido pelos casados com $39.8 \%$. Chamowicz (1998), considerando a situação conjugal dos idosos brasileiros, afirma que em 1993, a grande maioria das mulheres idosas 
(66.0\%) era composta por viúvas, solteiras ou separadas, contrastando com a situação dos homens de mesma idade ( $76.3 \%$ casados). Essa diferença se explica, não somente pela menor longevidade dos homens, mas, também, pela maior frequência dos homens se casarem novamente, após a viuvez, e maior tendência destes se casarem com mulheres mais jovens.

Contribuindo com o presente estudo, outros autores (Dishman \& Sallis, 1994; King et al., 1992) afirmam existir uma correlação positiva entre o grau de escolaridade e a adesão à atividade física (AF). Para os autores, indivíduos participantes de programas de AF preventiva apresentam um nível mais alto de educação formal do que aqueles que não participam. De acordo com Stephens e Caspersen (1994), estas atividades são consistentemente mais comuns entre grupos com maior nível de escolaridade. Os mesmos autores abordam que dados recentes de países como Austrália, Canadá e EUA revelam que grupos com maior grau de instrução são de 1.5 a 3.1 vezes mais ativos do que aqueles com menor grau, não sendo estes dados relacionados à faixa etária. O estudo de Rhodes et al. (1999) revelou que para indivíduos idosos, o grau de instrução foi positivamente correlacionado com a prática regular de AF.

O trabalho de Lima-Costa, Barreto e Giatti (2003), relacionado às condições de saúde e o uso de serviços de saúde da população brasileira, indicou que $69.0 \%$ dos idosos relatam ter pelo menos uma doença crónica, sendo a hipertensão e a artrite as mais comuns. A doença crónica é dominante e a maior causa de incapacidade, especialmente entre os mais velhos. Segundo Melzer e Parahyba (2004), a prevalência de incapacidade funcional na população idosa brasileira é maior em mulheres, aumenta com o avançar da idade e a disparidade económica e educacional são seus indicadores mais importantes.

Em relação ao questionário de qualidade de vida relacionada à saúde SF-36, as pontuações médias obtidas através da análise estatística ANOVA, revelou que não houve diferenças estatisticamente significativas entre os grupos $(p>.05)$. De acordo com a Tabela 1, o grupo PAF apresentou classificação "bom" nos domínios: Função Física, Rol Físico e Rol Emocional; o grupo NPAF apresentou classificação "bom" nos domínios: Função Física e Rol Emocional. Nos demais domínios, ambos os grupos encontraram-se na classificação "regular", porém em todos os itens, com exceção do domínio Dor Corporal, o grupo PAF apresentou escores mais altos quando comparados os grupo NPAF. Em estudo realizado também na Europa, com população idosa representativa da Espanha, foi observado que idosos envolvidos em atividades físicas apresentaram escores mais altos em praticamente todos os domínios do SF-36 (Guallar-Castillon et al., 2004).

Quanto à autonomia funcional de idosos, a análise estatística não destacou diferenças significativas entre os grupos $(p>.05)$. Porém, os resultados obtidos nesta pesquisa se assemelham a diferentes estudos (Aragão, Dantas, \& Dantas, 2003; César, Almeida, Pernambuco, Vale \& Dantas, 2004; Pernambuco, Vale, Baptista, Abreu \& Dantas, 2003; o grupo controle de Vale, 2004), onde a média dos grupos estudados foi considerada como "fraco" em todos os itens, com exceção do teste LCLC, que no estudo de César et al. (2004) obteve a classificação "muito bom". No teste $\mathrm{C} 10 \mathrm{M}$, em vários trabalhos (Geraldes, 2000; Vale, Aragão \& Dantas, 2003a; Vale et al., 2003b; Vale, 2004; Varejão, Dantas, \& Matsudo, 2007), os tempos obtidos foram classificados em "muito bom" e nos demais estudos os resultados classificaram-se nas pontuações intermediárias. As diferenças obtidas nos resultados se dão principalmente pelo tipo de intervenção aplicada às diferentes amostras estudadas. Por exemplo, um tempo satisfatório na execução deste teste é de grande importância para os idosos atravessarem uma rua de forma segura.

No teste LPS, o grupo controle do estudo de Vale (2004), o grupo avaliado no início do treinamento de Pernambuco et. al. (2003) e o 
grupo do estudo de Geraldes (2000) apresentaram tempos na classificação "fraco". Outros estudos (Baptista, Vale, Pernambuco, \& Dantas, 2003; Baptista, 2004; Vale et al., 2003a; Vale, 2004) apresentaram tempos de execução neste teste classificados como "muito bom". Os resultados das demais pesquisas encontraram-se nos outros níveis de classificação do padrão do protocolo GDLAM. Através do tempo gasto na execução deste teste pode-se ter uma noção da facilidade ou dificuldade que o idoso tem para levantar-se de uma cadeira, o que é um movimento de grande frequência nas atividades da vida diária.

No teste LPDV, apenas o trabalho de Vale et al. (2003b) apresentou o resultado num nível de classificação "muito bom", o que pode estar relacionado com o treinamento de força que foi utilizado na intervenção. Os outros estudos apresentaram as demais classificações.

No estudo de Geraldes (2000), este teste foi iniciado na posição de decúbito dorsal, diferentemente dos outros estudos. Esta alteração leva a um aumento no tempo de execução do mesmo, uma vez que o idoso, primeiramente, gira o corpo para a posição de decúbito ventral, para depois se levantar. Este teste representa o movimento de levantar-se do chão ou de uma cama.

O teste LCLC, foi menos utilizado nos estudos observados, porém é de grande importância como instrumento para se avaliar a agilidade e o equilíbrio do idoso. Nenhum estudo apresentou a classificação "muito bom", fazendo-se necessária a sua aplicação em mais pesquisas.

Como limitações deste estudo, destaca-se especialmente a amostra selecionada e o tipo de atividade física praticada pelas idosas. Houve um número pequeno de idosas e que, independentemente da prática de atividade física ou não, em sua maioria apresentava-se com alguma debilidade física, o que pode ter comprometido, de certa forma, os resultados desta pesquisa, não apresentando diferença significativa entre os grupos.

\section{CONCLUSÕES}

Após a análise dos resultados concluiu-se que as idosas praticantes de atividades físicas, embora tenham apresentado tendência de superioridade, apresentaram tempo de execução em todos os testes relacionados à capacidade funcional, similares ao de idosas não praticantes de atividade física, sendo todas classificadas como "fracas" de acordo com o protocolo GDLAM.

Quanto à medição da qualidade de vida relacionada à saúde, novamente nenhuma diferença entre as praticantes e não praticantes de atividade física foi observada, sendo que as praticantes de atividade física, da mesma forma, apresentaram uma tendência de escores mais alto quando comparados aos observados em idosas sedentárias. A falta de significância estatística, pode estar relacionada a variabilidade do grupo de não praticantes de atividade física.

Sugere-se para estudos futuros, uma análise mais detalhada do tipo de atividade física realizada, quanto ao tempo, frequência, intensidade e metodologia empregada, pois as atividades físicas realizadas de forma global nem sempre são suficientes para o idoso manter uma boa capacidade funcional.

\section{REFERÊNCIAS}

Alexander, N. B., Ulbrich, J., Raheja, A., \& Channer, D. (1997). Rising from the floors in older adults. Journal of the American Geriatrics Society, 45(5), 564-569.

Andreotti, R. A., \& Okuma, S. S. (1999). Validação de uma bateria de testes de atividades da vida diária para idosos fisicamente ativos. Revista Paulista de Educação Física, 13 (1), 46-66.

Aragão, J. C., Dantas, E. H., \& Dantas, B. H. (2003). Efeitos da resistência muscular localizada visando a autonomia funcional e a qualidade de vida do idoso. Fitness \& Performance Journal, 1 (3), 29-37.

Baptista, M. R. (2004). A prática do yoga sobre a autonomia funcional e qualidade de vida em mulheres senescentes. Dissertação de Mestrado em Ciência da Motricidade Humana, Universidade Castelo Branco, Rio de Janeiro, Brasil. 
Baptista, M. R., Vale, R. G., Pernambuco, C. S., \& Dantas, E. H. (2003). O yoga na autonomia funcional em mulheres senescentes. Comunicação apresentada no XXVI Simpósio Internacional de Ciências do Esporte, São Paulo, Brasil.

César, E. P., Almeida, O. V., Pernambuco, C. S., Vale, R. G., \& Dantas, E. H. (2004). Aplicação de quatro testes do protocolo GDLAM - Grupo de Desenvolvimento Latino-Americano Para Maturidade. Revista Mineira de Educação Física, 12(1), 18-37.

Chamowicz, F. (1998). Os idosos brasileiros no século XXI: Demografia, saúde e sociedade. Belo Horizonte: Postgraduate.

Ciconelli, R. M. (1997). Tradução para o português e validação do questionário genérico de avaliação de qualidade de vida "Medical Outcomes Study 36 - item short - Form Health Survey (SF-36). Tese de doutorado, Escola Paulista de Medicina de São Paulo, Universidade Federal de São Paulo, São Paulo, Brasil

Dantas, E. H., Vale, R. G., \& Pernambuco, C. S. (2004). Protocolo GDLAM de avaliação da autonomia funcional. Fitness \& Performance Journal, 3(3), 169-180.

Dishman, R. K., \& Sallis, J. F. (1994). Determinants and interventions for physical activity and exercise. In C. Bouchard, R. J. Shepard, \& T. Stephens (Eds.), Physical activity, fitness and health: International proceedings and consensus statement (pp. 215-238). Champaign, IL: Human Kinetics.

Geraldes, A. A. (2000). Efeitos do treinamento contra resistência sobre a força muscular e o desempenho de habilidades funcionais selecionadas em mulheres. Dissertação de Mestrado em Ciência da Motricidade Humana, Universidade Castelo Branco, Rio de Janeiro, Brasil.

Gonçalves, L. H., Dias, M. M., \& Liz, T. G. (1999). Qualidade de vida de idosos independentes segundo proposta de avaliação de Flanagan. Revista O Mundo da Saúde, 23 (4), 214-220.

Guallar-Castillon, P., Santa-Olalla, P. P., Banegas, J. R., Lopez, E., \& Rodriguez-Artalejo, F. (2004). Actividad física y calidad de vida de la población adulta mayor en España. Medicina Clínica, 123, 606-610.

Guralnik, J. M., Ferrucci, L., Pieper, C. F., Leveille, S. G., Markides, K. S., Ostir, G. V., ... Wallace, R. B. (2000). Lower extremity function and subsequent disability consistency across studies, predictive models and value of gait speed alone compared with the short physical performance battery. Journal of Gerontology, 55(4), 221-231.

Instituto Brasileiro de Geografia e Estatística - IBGE (2002). Perfil dos idosos responsáveis pelos domicílios. Consultado em 25 de Fevereiro de 2009, a partir de http://www.ibge.gov.br/ho me/presidencia/noticias/25072002pidoso.shtm

King, A. C., Blair, S. N., Bild, D. E., Dishman, R. K., Dubbert, P. M., Marcus, B. H., ... Yeager, K. K. (1992). Determinants of physical activity and interventions in adults. Medicine $\mathcal{E}$ Science in Sports \& Exercise, 24(6), S221-236.

Lima-Costa, M. F., Barreto, S. M. \& Giatti, L. (2003). Condições de saúde, capacidade funcional, uso de serviços de saúde e gastos com medicamentos da população idosa brasileira: Um estudo descritivo baseado na Pesquisa Nacional por Amostra de Domicílios. Cadernos de Saúde Pública, 19(3), 735-743.

Matsudo, S. M. (2002). Envelhecimento, atividade física e saúde. Revista Mineira de Educação Física, 10(1), 193-207.

Mazo, G. Z., Lopes, M. A., \& Benedetti, T. B. (2004). Atividade física e o idoso: Concepção gerontológica (2a ed.). Porto Alegre: Sulina.

Melzer, D., \& Parahyba, M. I. (2004). Sociodemografic correlates of mobility disability in older brazilians: Results of the first national survey. Age and Ageing, 33 (3), 253-257.

Nahas, M. V. (2006). Atividade física, saúde e qualidade de vida: Conceitos e sugestões para um estilo de vida ativo ( $4^{\mathrm{a}}$ ed.). Londrina: Midiograf.

Netto, M., \& Ponte, J. R. (2005). Envelhecimento: Desafio na transição do século. In M. P. Netto (Ed.), Gerontologia (pp. 3-12). São Paulo: Editora Atheneu.

Okuma, S. S. (1998). O idoso e a atividade física: Fundamentos e pesquisa. Campinas: Papirus Editora.

Pernambuco, C. S., Vale, R. G., Baptista, M. R., Abreu, F. M., \& Dantas, E. H. (2003). Perfil da autonomia funcional de idosos no ingresso de um programa de shiatsuterapia no município de Araruama. Comunicação apresentada no XXVI Simpósio Internacional de Ciências do Esporte, São Paulo, Brasil.

Rhodes, R. E., Martin, A. D., Tauton, J. E., Rhodes, E. C., Donnelly, M., \& Elliot, J. (1999). Factors associated with exercise adherence among older adults: An individual perspective. Sports Medicine, 28(6), 397-411. 
Santos, S. C., \& Knijnik, J. F. (2006). Motivos de adesão à prática de atividade física na vida adulta intermediária. Revista Mackenzie de Educação Física, 5(1), 23-34.

Santos, S. R., Santos, I. B., Fernandes, M. G., \& Henriques, M. E. (2002). Qualidade de vida do idoso na comunidade: Aplicação da escala de Flanagan. Revista Latino-Americana de Enfermagem, 10(6), 757-764.

Sipila, S., Multanen, J., Kallinen, M., Era, P., \& Suominen, H. (1996). Effects of strength and endurance training on isometric muscle strength and walking speed in elderly women. Acta Physiologica Scandinavica, 156, 457-464.

Stephens, T., \& Caspersen, C. J. (1994). The demography of physical activity. In C. Bouchard, R. J. Shepard, \& T. Stephens (Eds.), Physical activity, fitness and health: International proceedings and consensus statement (pp. 205-213). Champaign, IL: Human Kinetics.

Unitat de Recerca en Serveis Sanitaris - URSS (2000). Manual de puntuación de la versión española del Cuestionario de Salud SF-36. Barcelona: Institut Municipal d'Investigació Mèdica.
Vale, R. G. (2004). Efeitos do treinamento de força e de flexibilidade sobre a autonomia e qualidade de vida de mulheres senescentes. Dissertação de Mestrado em Ciência da Motricidade Humana, Universidade Castelo Branco, Rio de Janeiro, Brasil.

Vale, R. G., Aragão, J. C., \& Dantas, E. H. (2003a). A flexibilidade na autonomia funcional de idosas independentes. Fitness \& Performance Journal, 2(1), 23-29.

Vale, R. G., Baptista, M. R., Pernambuco, C. S., Vieira, F. R., Aragão, J. C., Damasceno, V., ... Dantas, E. H. (2003b). Treinamento resistido de força em idosas independentes. Comunicação apresentada no XXVI Simpósio Internacional de Ciências do Esporte, São Paulo, Brasil.

Varejão, R. V., Dantas, E. H., \& Matsudo, S. M. (2007). Comparação dos efeitos do alongamento e do flexionamento, ambos passivos, sobre os níveis de flexibilidade, capacidade funcional e qualidade de vida do idoso. Revista Brasileira de Ciência e Movimento, 15(2), 87-95.

(c))EY-Nc Todo o conteúdo da revista Motricidade está licenciado sob a Creative Commons, excepto quando especificado em contrário e nos conteúdos retirados de outras fontes bibliográficas. 